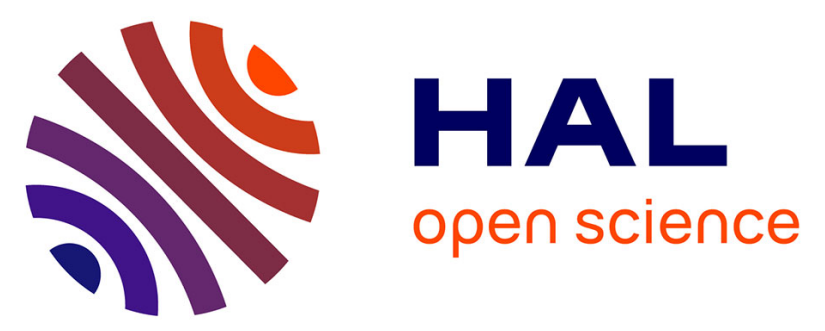

\title{
Direct Mechanical Detection and Measurement of Wave-Matter Orbital Angular Momentum Transfer by Nondissipative Vortex Mode Conversion
}

B Sanchez-Padilla, L Jonusauskas, M. Malinauskas, R. Wunenburger, E. Brasselet

\section{To cite this version:}

B Sanchez-Padilla, L Jonusauskas, M. Malinauskas, R. Wunenburger, E. Brasselet. Direct Mechanical Detection and Measurement of Wave-Matter Orbital Angular Momentum Transfer by Nondissipative Vortex Mode Conversion. Physical Review Letters, 2019, 123, 10.1103/PhysRevLett.123.244301. hal-02454448

\section{HAL Id: hal-02454448 \\ https://hal.science/hal-02454448}

Submitted on 24 Jan 2020

HAL is a multi-disciplinary open access archive for the deposit and dissemination of scientific research documents, whether they are published or not. The documents may come from teaching and research institutions in France or abroad, or from public or private research centers.
L'archive ouverte pluridisciplinaire HAL, est destinée au dépôt et à la diffusion de documents scientifiques de niveau recherche, publiés ou non, émanant des établissements d'enseignement et de recherche français ou étrangers, des laboratoires publics ou privés. 


\title{
Direct Mechanical Detection and Measurement of Wave-Matter Orbital Angular Momentum Transfer by Nondissipative Vortex Mode Conversion
}

\author{
B. Sanchez-Padilla $\odot,{ }^{1}$ L. Jonusauskas, ${ }^{2}$ M. Malinauskas $\odot,{ }^{2}$ R. Wunenburger, ${ }^{3}$ and E. Brasselet ${ }^{1, *}$ \\ ${ }^{1}$ University of Bordeaux, CNRS, LOMA, UMR 5798, F-33400 Talence, France \\ ${ }^{2}$ Laser Research Center, Physics Faculty, Vilnius University, Saulètekio Ave. 10, LT-10223 Vilnius, Lithuania \\ ${ }^{3}$ Sorbonne Université, CNRS, Institut Jean le Rond d'Alembert, F-75005 Paris, France
}

(Received 16 July 2019; published 9 December 2019)

\begin{abstract}
We quantitatively report on the rotational mechanical effect of wave orbital angular momentum on matter by nondissipative vortex mode conversion. Our experiments consist of ultrasonic waves reflected off freely spinning helical acoustic mirrors that are capillary trapped at a curved air-water interface. Considering helical mirrors with integer topological charges these results represent the demonstration of the experiment proposed by Allen et al. originally introduced in the optical domain [Phys. Rev. A 45, 8185 (1992)], whose quantitative implementation remains elusive to date whatever the nature of the wave. The study is further generalized to helical mirrors with fractional charges.
\end{abstract}

DOI: 10.1103/PhysRevLett.123.244301

It took three centuries to turn Kepler's intuition that sunlight pushes away the tail of a comet [1] into documented experimental facts [2,3]. Noticeably, radiation forces are not the privilege of electromagnetism and strong analogies with acoustics have been emphasized in several works despite the distinct nature (transverse vs longitudinal) of these waves, as discussed in the context of fluid interfaces deformations [4] and tweezers [5]. The analogy also applies to radiation torques when considering beams carrying on-axis phase singularity (i.e., vortex beams). The complex amplitude $A$ of a vortex beam has an azimuthal dependence of the form $A \propto \exp (i \ell \phi)$, where $\ell$ integer is the topological charge and $\phi$ is the azimuthal angle in the transverse plane. In particular, the ratio of the orbital angular momentum flux to energy flux of vortex beams has been predicted to be $\ell / \omega$, where $\omega$ is the angular frequency of the wave, originally in the paraxial limit in optics [6] and in acoustics [7].

Inspired by Beth, who mechanically assessed the spin angular momentum of light [8], Allen et al. proposed an orbital analog experiment via a lossless interaction between optical vortices and matter [6]. Since then, numerous experimental works dealing with orbital angular momentum transfer have been made. We refer to Refs. [9-12] as a nonexhaustive set of earlier works in optics while much fewer studies have been reported in acoustics [13-21]. To date, however, there is no experiment dedicated to the quantitative assessment of the direct mechanical effect of wave orbital angular momentum on matter by lossless vortex mode conversion, whatever the nature of the wave. This represents the primary motivation of this work. Noteworthy, by doing so, we report on a heating-free route to contactless acoustic rotational manipulation of matter. Moreover, recalling that at equal power level, acoustic torques are larger than optical ones by a typical factor $10^{8}-10^{9}$ in the $0.1-1 \mathrm{MHz}$ frequency range [16], we envision that ultrasound-induced torque will lead to the advent of vortex echography, which might revolutionize medical imaging.

To our knowledge, only two experimental attempts have been reported so far. In 2005, Beijersbergen and Woerdman implemented the original optical proposal by Allen et al., however concluding negatively [22]. Ten years later, Wunenburger et al. suggested the use of acoustic chiral scattering as a nondissipative process [23]. However, the poor quality of the fabricated scatterers and the used transmissive approach prevented obtaining both pure vortex mode scattering and quantitative measurements due to dissipative drawbacks as the wave propagates through the scatterer. Here we solve the problem by proposing a virtually nondissipative approach. This is done by using an airborne ultrasonic wave at $f=100 \mathrm{kHz}$ frequency impinging on a solid material, which ensures a strong acoustic impedance mismatch. Towards a quantitative assessment, let us consider the air-matter reflectivity coefficient $\mathcal{R}=\left|\left(\rho_{1} \kappa_{0}-\rho_{0} \kappa_{1}\right) /\left(\rho_{1} \kappa_{0}+\rho_{0} \kappa_{1}\right)\right|^{2}$, where $\rho_{j}$ is the density and $\kappa_{j}=k_{j}+i \alpha_{j}$ is the complex wave vector with $k_{j}=2 \pi f / c_{j} ; c_{j}$ and $\alpha_{j}$ being the sound wave celerity and attenuation. The indices $j=(0,1)$ refer to air and matter, with on the one hand, $\rho_{0}=1.2 \mathrm{~kg} \mathrm{~m}^{-3}$, $c_{0}=343 \mathrm{~m} \mathrm{~s}^{-1}$, and $\alpha_{0} \simeq 0.38 \mathrm{~m}^{-1}$ [24] and, on the other hand, $\rho_{1}=1.16 \pm 0.04 \mathrm{~kg} \mathrm{~m}^{-3}$ for our material, for which we measure $c_{1} \simeq 2.3 \mathrm{~km} \mathrm{~s}^{-1}$ and $\alpha_{1} \simeq 10 \mathrm{~m}^{-1}$ from the acoustic pulse transmission technique in water [25]. This gives a virtually pure reflectivity, $\mathcal{R}=1$, whatever is $\alpha_{1}$.

The chiral scatterers are helical mirrors that consist of $R=15 \mathrm{~mm}$-radius 3D printed spiral-shaped plates made of prototyping resin PR57-K (Autodesk) and designed with $\ell$ 


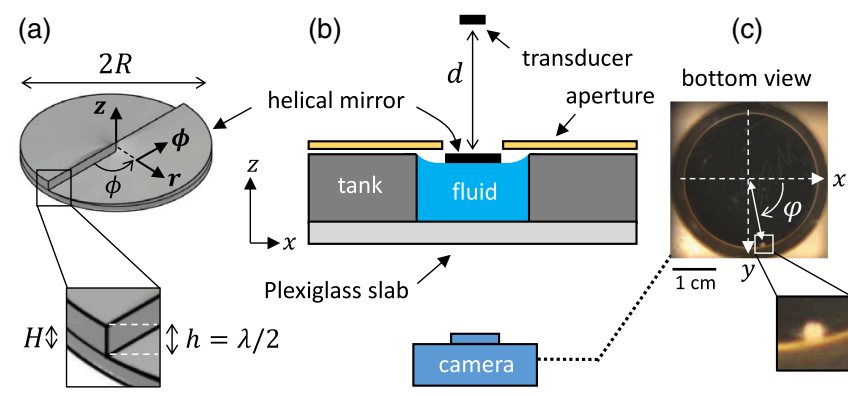

FIG. 1. Acoustic radiation torque experiment. (a) Designed helical mirror, here with $\ell=2$, with $(\mathbf{r}, \boldsymbol{\phi}, \mathbf{z})$ the unit vectors of the cylindrical basis. (b) Sketch of the setup, where the camera is used to record the rotational dynamics of the helical mirror from its flat side. (c) In situ bottom view of a helical mirror, where the printed marker is on the back side of the mirror used to monitor its angular dynamics $\varphi(t)$.

identical adjacent angular sectors having azimuthally varying thickness $e(\phi)=\ell \lambda \phi /(4 \pi)$, on the top of a $H=1 \mathrm{~mm}$-thick disk pedestal, where $\lambda=3.44 \mathrm{~mm}$ is the acoustic wavelength in the air, see Fig. 1(a). The step height $h=\lambda / 2$ ensures that a phase profile $\exp \left(i \ell^{\prime} \phi\right)$ is imparted to the reflected wave propagating towards $z>0$ [26], with $\ell^{\prime}=-\ell$. An acoustic torque $\Gamma \mathbf{z}=(\ell P / \omega) \mathbf{z}$ is thus exerted on the mirror, where $\mathbf{z}$ is the unit vector pointing towards the transducer and $P$ is the intercepted power by the helical mirror.

The setup is depicted in Fig. 1(b) and all experiments are made at room temperature, humidity, and pressure. We use a cylindrical polyethylene tank with $25 \mathrm{~mm}$ height and $37 \mathrm{~mm}$ diameter, whose bottom part is closed by a $5 \mathrm{~mm}$ thick Plexiglass slab through which the rotational dynamics of the mirror is video recorded. The tank is initially fully filled with an aqueous solution with 65 wt \% glycerol, on the top of which is deposited the helical mirror, flat side down [27]. Then we remove a sufficient volume of fluid with a syringe in order to obtained a curved air-fluid interface while keeping the triple contact line attached to the circular upper edge of the tank. This preparation protocol is both robust and reproducible. Capillary effects thus ensure self-centering of the mirror while it remains free to rotate owing to the fluid environment. The acoustic source is a flat transducer with a $10 \mathrm{~mm}$ diameter circular cross section placed at a distance $d=100 \mathrm{~mm}$ from the mirror. Its output power $P_{0}$ is set to a constant value during all the experiments and we measure $P_{0} \simeq 24 \mathrm{~mW}$ from a radiation pressure experiment. Namely, we irradiate from the air a precision scale (which behaves as an acoustic mirror) at $45^{\circ}$ incidence and the measured mass $m_{0}$ is related to $P_{0}$ via $m_{0} g=2 \cos (\pi / 4) P_{0} / c_{0}$, where $g$ is the acceleration of gravity and $c$ the sound speed in the air. Onaxis centering of the transducer and the mirror is ensured owing to a $1 \mathrm{~mm}$ diameter hole in the center of the transducer, through which the outgoing spot of a halogen lamp allows reproducible and accurate positioning. Also, a flat mirror with a $32 \mathrm{~mm}$ diameter circular aperture is placed on the top of the tank, see Fig. 1(b), in order to prevent wave reflection off the curved water interface to reach the transducer.

The choice $d=100 \mathrm{~mm}$ results from a practical tradeoff between (i) maximizing the power $P$ intercepted by the helical mirror (which favors choosing small distances) and (ii) minimizing Fabry-Pérot cavity effects between the mirror and the transducer (which favors choosing large distances). This is illustrated in Figs. 2(a) and 2(b) which, respectively, display the dependance of $P$ on the distance $d$ and the corresponding power $P^{\prime}$ of the reflected field intercepted by the transducer. The calculations are made by using the scalar beam propagation method based on two-dimensional fast Fourier transform. Namely, omitting the time oscillating phase factor exp $(-i \omega t)$, the transverse field amplitude at $z=z_{2}$ is evaluated from the field at $z=z_{1}$, according to $A\left(x, y, z_{2}\right)=$ $\mathcal{F}^{-1}\left\{\exp \left[\left(i k_{z}-\alpha_{0} k / k_{z}\right)\left(z_{2}-z_{1}\right)\right] \mathcal{F}\left[A\left(x, y, z_{1}\right)\right]\right\}$. Here, $\mathcal{F}\left(\mathcal{F}^{-1}\right)$ refers to $2 \mathrm{D}$ (inverse) Fourier transform and $\exp \left[\left(i k_{z}-\alpha_{0} k / k_{z}\right) z\right]$ is the complex propagation operator in the Fourier domain with $k_{z}=\left(k^{2}-k_{x}^{2}-k_{y}^{2}\right)^{1 / 2}$. The transducer is modeled as a uniform disk source having a
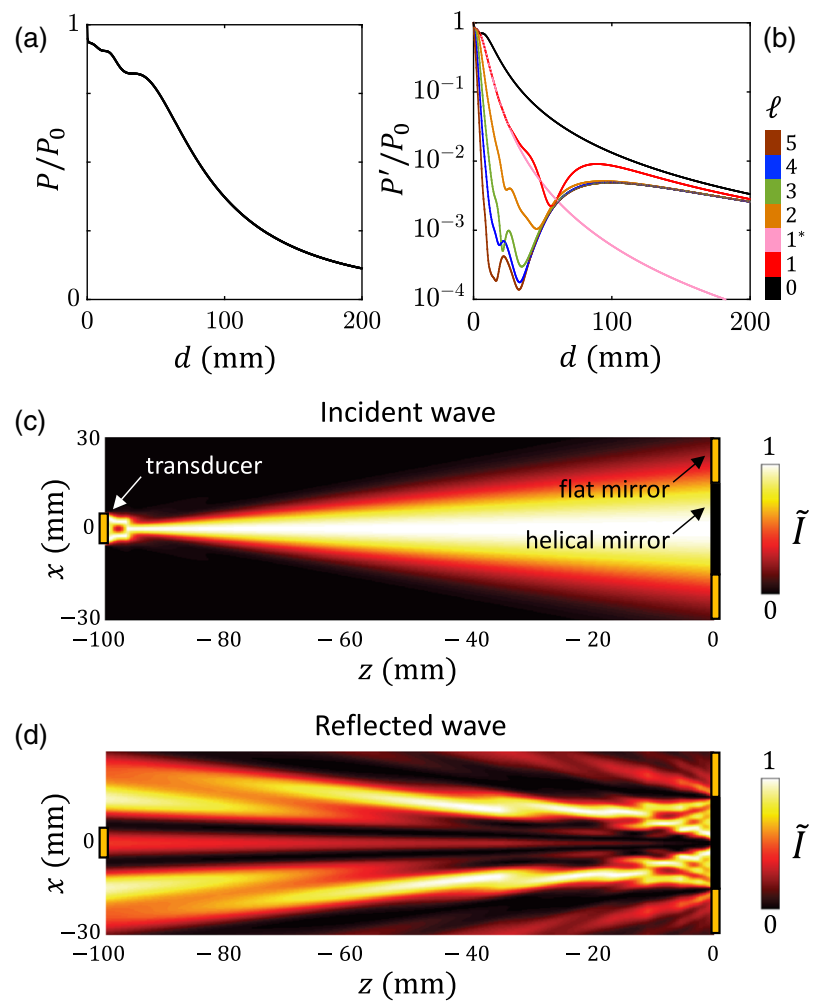

FIG. 2. (a) Power $P$ intercepted by the helical mirror as a function of its distance $d$ from the transducer. (b) Corresponding power $P^{\prime}$ of the reflected field intercepted back by the transducer for $0 \leq \ell \leq 5$. Also, $\ell=1^{*}$ refers to a helical mirror with unit topological charge and infinite extent. Both $P$ and $P^{\prime}$ are normalized to the total output power $P_{0}$ of the transducer. (c) Normalized intensity distribution of the incident wave in the meridional plane $(x, z)$ as a function of $z$ for $d=100 \mathrm{~mm}$. (d) Same as in panel (c) for the reflected field with $\ell=2$. 
uniform displacement amplitude $A_{0}$ and the outer flat mirror defines $z=0$. In addition, the helical mirror is modeled by a pure phase reflective mask described by the amplitude reflection function $\exp \left(i \ell^{\prime} \phi\right)$.

The $(x, z)$ meridional cross sections of the calculated normalized intensity distribution $\tilde{I}(x, z)=|A(x, 0, z)|^{2} /$ $\max _{(x, y)}\left[|A(x, y, z)|^{2}\right]$ of the incident and reflected field for $d=100 \mathrm{~mm}$, are shown in Figs. 2(c) and 2(d). In particular, Fig. 2(d) allows qualitative understanding of the dependence of $P^{\prime}$ on $\ell$ displayed in Fig. 2(b). Indeed, the doughnut intensity profile of the reflected vortex wave leads to a drastic reduction at short distance $(d \lesssim 10 \mathrm{~mm})$ of the cross section of the field with the transducer when using a helical mirror $(\ell \neq 0)$ instead of a flat one $(\ell=0)$. This effect is strengthened as $\ell$ increases as expected from the fact that the area of the vortex core region increases with $|\ell|$. For intermediate distance $(10 \lesssim d \lesssim 100 \mathrm{~mm})$, interference between the field contributions from the flat mirror and the helical mirror lead to nonmonotonic behavior. This is supported by the case $\ell=1^{*}$ referring to the helical mirror with infinite extent, which exhibits no power oscillation vs $d$. Finally, the behavior at large distance $(d \gtrsim 100 \mathrm{~mm})$ is dictated by the reflexion from the outer flat mirror leading to nonzero on-axis intensity, see Fig. 2(d), which leads to an asymptotic behavior independent on $\ell$ [Fig. 2(b)].

Spinning experiments are monitored by tracking the position of a marker printed on the back side of the mirror, see Fig. 1(c). The typical steady circular trajectory, see Fig. 3(a), points out the quality of the on-axis alignment of the tank, the rotating element and the transducer. In addition, the long-term linear dependence of the azimuthal angle $\varphi$ that refers to the angular position of the marker, see Fig. 3(b), emphasizes the stationarity of the experiment. The latter behavior is used to measure the steady angular frequency $\langle\Omega\rangle$.

With the aim at measuring the acoustic torque, we rely on the equation of motion of the rotating element whose time-dependent angular frequency dynamics $\Omega(t)$ is
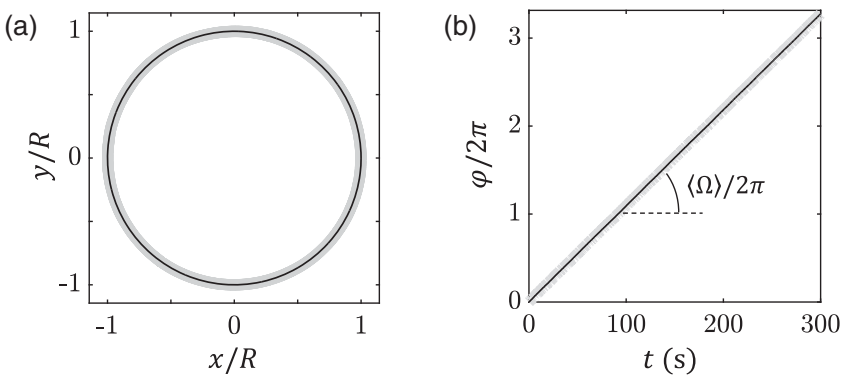

FIG. 3. (a) Typical trajectory, in the $(x, y)$ plane, of the marker at the backside of the spinning helical mirror over $\sim 3$ full turns. (b) Experimental dynamics of the azimuthal angular coordinate of the marker $\varphi$, which is evaluated from the trajectory shown in panel (a), taking $\varphi=0$ at $t=0$. Data refer to an $\ell=5$ helical mirror. Thick gray curve: experimental data. Thin dark circle: best circular (a) and linear (b) fit.
$J(d \Omega / d t)=\Gamma-C \Omega$ with $J=J_{\text {mirror }}+J_{\text {seat }}$ the moment of inertia of the object "mirror + seat" [27], where $C \Omega$ is the viscous drag torque and $C>0$ the viscous drag coefficient. Even at steady state, $C$ depends a priori on $\Omega$ due to inertial contributions to the viscous drag torque exerted on the rotating object. However, for small enough Reynolds number $\operatorname{Re}=\Omega R^{\prime 2} \rho / \eta$ [27], with $\rho \simeq$ $1170 \mathrm{~kg} \mathrm{~m}^{-3}$ the mass density of the fluid and $\eta \simeq$ $15 \mathrm{mPa}$ s its dynamic viscosity, $C$ can be assumed constant (i.e., Stokes hydrodynamic approximation). In the present case, the maximal angular frequency corresponds to the data shown in Fig. 3 and is up to $0.07 \mathrm{rad} \mathrm{s}^{-1}$, which gives $\operatorname{Re} \sim 1$. In order to evaluate how well the Stokes approximation is satisfied, we estimate the first-order correction to the viscous drag torque due to inertial effects according to Ref. [28] that deals with an infinitely thin disk rotating at the constant rotation rate $\Omega$ in a unbounded fluid. Namely, the inertial correction can be neglected when $\epsilon \operatorname{Re}^{2} \ll 1$ where $\epsilon \sim 10^{-3}$, which is satisfied in our experiments.

The measurement of the acoustic torque accounts that steady spinning dynamics implies measuring the viscous constant $C$, which has the advantage of not requiring a hydrodynamical model. This is done by studying the relaxation dynamics once the irradiation is turned off at $t=t_{0}$. Indeed, Eq. (1) gives $\Omega\left(t>t_{0}\right)=\Omega\left(t_{0}\right) \exp [-(t-$ $\left.\left.t_{0}\right) / \tau\right]$ with $\tau=J / C$ the characteristic relaxation time. Practically, we directly measure the angular displacement $\varphi(t)$, whose relaxation dynamics is expressed as $\varphi\left(t>t_{0}\right)=\varphi\left(t_{0}\right)+\Omega\left(t_{0}\right) \tau\left\{1-\exp \left[-\left(t-t_{0}\right) / \tau\right]\right\}$. We evaluate $C$ by fitting the data over $1 \mathrm{~s}$ duration using $\Omega\left(t_{0}\right)$ and $\tau$ as adjustable parameters, noting that $J$ is measured independently, see thin solid curve in Fig. 4. Following this protocol, we study the dependence of the acoustic torque on the topological charge of the helical mirror. This is done by performing five independent experiments for each value of $\ell$ from 1 to 5, for two distinct seats [29]. For each experiment, the helical mirror is removed and replaced according to the protocol described earlier, and we record both the steady state and the relaxation dynamics from

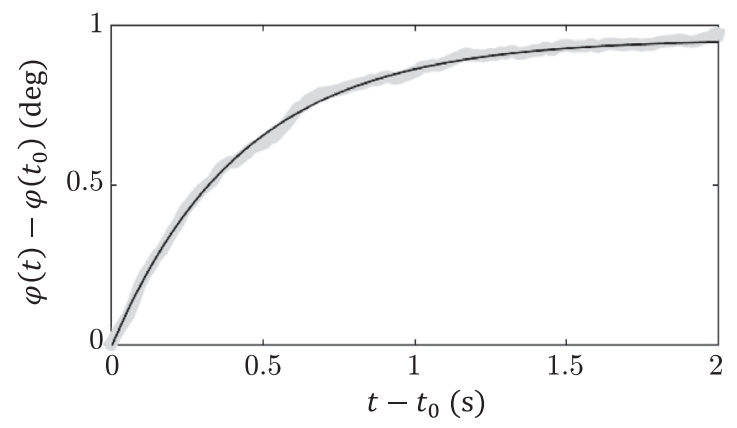

FIG. 4. Typical relaxation dynamics of the angular position of the helical mirror, where $t=t_{0}$ refers to the time at which the acoustic source is turned off. Thick gray curve: experimental data. Thin dark curve: fit giving access to the ratio $C / J$. Data refer to an $\ell=5$ helical mirror. 

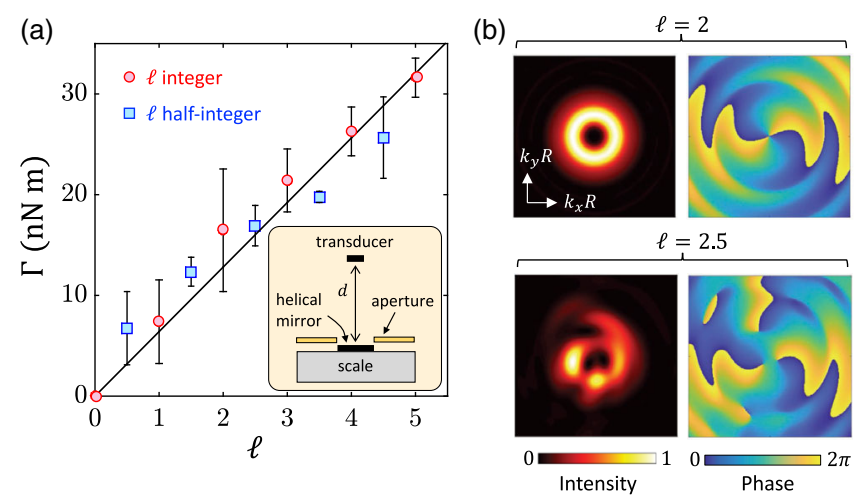

FIG. 5. (a) Acoustic torque as a function of its topological charge. Markers: experimental data. Solid line: linear fit. Inset refers to radiation force balance measurement.(b) Calculated farfield normalized intensity and phase distribution of the wave reflected off the helical mirror for $\ell=2$ and 2.5. Panels refers to the range $-5 \leq\left(k_{x} R, k_{y} R\right) \leq 5$.

which $C,\langle\Omega\rangle$, and $\Omega\left(t_{0}\right)$ are all measured. Since the steady rotation and the relaxation dynamics are associated with distinct irradiation conditions, we evaluate the acoustic torque from irradiation-free quantities, namely, $\Gamma=C \Omega\left(t_{0}\right)$, noting that $\Omega\left(t_{0}\right) /\langle\Omega\rangle=1.08 \pm 0.18$. The results of these 50 independent experiments are shown as circle markers in Fig. 5(a), where each marker refers to the average over 10 measurements and error bars refer to the corresponding standard deviation.

The results are generalized to half-integer topological charges $\ell=n-1 / 2$ with $n$ integer from 1 to 5 , which is done by using a $n$-step design with $n$-dependent step heights $h_{n}=(\lambda / 2)[1-(1 / 2 n)]$. Here again, five independent experiments are performed for each value of $\ell$. The corresponding results of these 25 independent additional experiments are shown as square markers in Fig. 5(a). Noteworthy, noninteger values of $\ell$ do not correspond to the case of pure vortex mode conversion as originally discussed by Allen et al. [6]. This is illustrated in Fig. 5(b), which displays the calculated far-field intensity and phase distributions of the wave reflected off the helical mirror for $\ell=2$ and 2.5, discarding the outer contribution reflected off the outer flat mirror. Indeed, axisymmetric doughnutshaped intensity profile and on-axis phase singularity with topological charge $\ell^{\prime}=-\ell$ is obtained for integer $\ell$ while a constellation of singularities with charges \pm 1 emerges otherwise [30].

Quantitative agreement is assessed recalling the expression $\Gamma=\ell P / \omega$, which holds for noninteger values of $\ell$ as well. The linear fitting of the data displayed in Fig. 5(a) using $P$ as the single adjustable parameter gives $P=4.0 \mathrm{~mW}$. This value is compared to that obtained from a radiation force balance measurement made using a precision scale in the same conditions as used in the acoustic torque experiments, see inset of Fig. 5(a). The mass $m$ associated with the acoustic radiation pressure exerted on a flat mirror $(\ell=0)$ is measured and we find $m=5.1 \pm 0.2 \mathrm{mg}$, from which we deduce a power $P=m g c_{0} / 2=7.5 \pm 0.3 \mathrm{~mW}$.

The discrepancy between the power $P$ evaluated from the acoustic torque and force experiments calls for several comments. First, we note that the possibility that the transducer emits power at frequencies other than $100 \mathrm{kHz}$ is neither affecting the acoustic radiation force, which does not depend on the frequency, nor the acoustic radiation torque since the direct scaling $\Gamma \propto \omega^{-1}$ is compensated by the fact that $\ell \propto \lambda^{-1} \propto \omega$. Second, we recall that used expression $\Gamma=\ell P / \omega$ is only valid in the paraxial approximation while any helical mirror a priori does not behave in a paraxial manner in its center as the spiral slope angle $\beta(r)=$ $\arctan [\ell h /(2 \pi r)]$ diverges as $r \rightarrow 0, r$ being the distance from the center. A quantitative estimation of its impact on the total torque exerted on a helical mirror is obtained from the basics of continuum mechanics, see Ref. [23], which gives $\Gamma=(4 \pi / c) \int_{0}^{D / 2} I(r) \sin \beta(r) \cos ^{2} \beta(r) r^{2} d r$. Here, accounting for the acoustic intensity profile given by simulations, we predict that our settings only give up to $\sim 7 \%$ underestimate of the power $P$ with respect to the paraxial framework. Third, the nonideality of the fabricated helical mirror, whose height is not independent on $r$ in practice, is another source of power mismatch. We estimate from the topographic inspection of the helical mirror that the nonuniform region has a maximal radius $r_{0} \sim 1 \mathrm{~mm}$. Since this corresponds to a $\sim 1 \%$ area of the helical mirror, we fairly conclude that the latter imperfections are not likely to bring an explanation. Fourth, we note that the effect of the acoustic rotational streaming [16], which corresponds here to a rotating airflow induced by the dissipation of the reflected acoustic vortex beam in the air, can be safely neglected here since the main source of the viscous torque comes from the liquid due to a large contrast of viscosity $\left(\eta_{\text {liquid }} / \eta_{\text {air }} \sim 7 \times 10^{2}\right)$. These comments being said, we admit that we do not have a definitive explanation for the observed acoustic power mismatch.

Summarizing, we have reported the direct mechanical detection and measurement of wave-matter orbital angular momentum transfer by vortex mode conversion. This represents the quantitative demonstration of the experiment proposed by Allen et al. in the optical domain in 1992 [6]. In addition, our experiments open for further quantitative acoustomechanical studies in gases, such as acoustic rotational streaming [16], which remains barely studied whatever the nature of the propagating medium despite its crucial role in acoustic vortex tweezers [19]. This also motivates the experimental exploration of recently unveiled wave features of inhomogeneous sound waves, namely, the existence of acoustic spin of surface waves [31] and progressive vortex beams [32].

The research leading to these results has received funding from CONACYT Mexico and LASERLABEUROPE (Grant Agreement No. 654148, European Union's Horizon 2020 research and innovation programme). 
*etienne.brasselet@u-bordeaux.fr

[1] J. Kepler, De cometis libelli tres (Augustae Vindelicorum, Augsburg, 1619).

[2] P. N. Lebedev, Experimental examination of light pressure, Ann. Phys. (Leipzig) 6, 433 (1901).

[3] E. F. Nichols and G. F. Hull, A preliminary communication on the pressure of heat and light radiation, Phys. Rev. (Series I) 13, 307 (1901); The pressure due to radiation. (Second Paper.), Phys. Rev. (Series I) 17, 26 (1903).

[4] N. Bertin, H. Chraibi, R. Wunenburger, J.-P. Delville, and E. Brasselet, Universal Morphologies of Fluid Interfaces Deformed by the Radiation Pressure of Acoustic or Electromagnetic Waves, Phys. Rev. Lett. 109, 244304 (2012).

[5] J.-L. Thomas, R. Marchiano, and D. Baresch, Acoustical and optical radiation pressure and the development of single beam acoustical tweezers, J. Quant. Spectrosc. Radiat. Transfer 195, 55 (2017).

[6] L. Allen, M. W. Beijersbergen, R. J. C. Spreeuw, and J. P. Woerdman, Orbital angular momentum of light and the transformation of laguerre-gaussian laser modes, Phys. Rev. A 45, 8185 (1992).

[7] B. T. Hefner and P. L. Marston, An acoustical helicoidal wave transducer with applications for the alignment of ultrasonic and underwater systems, J. Acoust. Soc. Am. 106, 3313 (1999).

[8] R. A. Beth, Mechanical detection and measurement of the angular momentum of light, Phys. Rev. 50, 115 (1936).

[9] H. He, M. E. J. Friese, N. R. Heckenberg, and H. Rubinsztein-Dunlop, Direct Observation of Transfer of Angular Momentum to Absorptive Particles from a Laser Beam with a Phase Singularity, Phys. Rev. Lett. 75, 826 (1995).

[10] M. E. J. Friese, J. Enger, H. Rubinsztein-Dunlop, and N. R. Heckenberg, Optical angular-momentum transfer to trapped absorbing particles, Phys. Rev. A 54, 1593 (1996).

[11] N. B. Simpson, K. Dholakia, L. Allen, and M. J. Padgett, Mechanical equivalence of spin and orbital angular momentum of light: An optical spanner, Opt. Lett. 22, 52 (1997).

[12] V. Garcés-Chávez, K. Volke-Sepulveda, S. Chávez-Cerda, W. Sibbett, and K. Dholakia, Transfer of orbital angular momentum to an optically trapped low-index particle, Phys. Rev. A 66, 063402 (2002).

[13] K. Volke-Sepulveda, A. O. Santillan, and R. R. Boullosa, Transfer of Angular Momentum to Matter from Acoustical Vortices in Free Space, Phys. Rev. Lett. 100, 024302 (2008).

[14] K. D. Skeldon, C. Wilson, M. Edgar, and M. J. Padgett, An acoustic spanner and its associated rotational doppler shift, New J. Phys. 10, 013018 (2008).

[15] C. E. M. Demore, Z. Yang, A. Volovick, S. Cochran, M. P. MacDonald, and G. C. Spalding, Mechanical Evidence of the Orbital Angular Momentum to Energy Ratio of Vortex Beams, Phys. Rev. Lett. 108, 194301 (2012).

[16] A. Anhauser, R. Wunenburger, and E. Brasselet, Acoustic Rotational Manipulation Using Orbital Angular Momentum Transfer, Phys. Rev. Lett. 109, 034301 (2012).

[17] Y. Li, G. Guo, J. Tu, Q. Ma, X. Guo, D. Zhang, and O. A. Sapozhnikov, Acoustic radiation torque of an acoustic-vortex spanner exerted on axisymmetric objects, Appl. Phys. Lett. 112, 254101 (2018).

[18] W. Li, M. Ke, S. Peng, F. Liu, C. Qiu, and Z. Liu, Rotational Manipulation by acoustic radiation torque of high-order vortex beams generated by an artificial structured plate, Appl. Phys. Lett. 113, 051902 (2018).

[19] D. Baresch, J.-L. Thomas, and R. Marchiano, Orbital Angular Momentum Transfer to Stably Trapped Elastic Particles in Acoustical Vortex Beams, Phys. Rev. Lett. 121, 074301 (2018).

[20] M. E. Terzi, S. A. Tsysar, P. V. Yuldashev, M. M. Karzova, and O. A. Sapozhnikov, Generation of a vortex ultrasonic beam with a phase plate with an angular dependence of the thickness, Moscow Univ. Phys. Bull. 72, 61 (2017).

[21] A. Marzo, M. Caleap, and B. W. Drinkwater, Acoustic Virtual Vortices with Tunable Orbital Angular Momentum for Trapping of Mie Particles, Phys. Rev. Lett. 120, 044301 (2018).

[22] M. W. Beijersbergen and J. Woerdman, Measuring orbital angular momentum of light with a torsion pendulum, in Proc. SPIE 5736, Nanomanipulation with Light (International Society for Optics and Photonics, 2005), Vol. 5736, pp. 111-126, https://doi.org/10.1117/12.584515.

[23] R. Wunenburger, J. I. V. Lozano, and E. Brasselet, Acoustic orbital angular momentum transfer to matter by chiral scattering, New J. Phys. 17, 103022 (2015).

[24] H. E. Bass, L.C. Sutherland, A. J. Zuckerwar, D. T. Blackstock, and D. Hester, Atmospheric absorption of sound: Further developments, J. Acoust. Soc. Am. 97, 680 (1995).

[25] H. Jeong and D. K. Hsu, Experimental analysis of porosityinduced ultrasonic attenuation and velocity change in carbon composites, Ultrasonics 33, 195 (1995).

[26] We adopt the convention of a propagating phase term exp $(-i \omega t \pm i k z)$ for a wave propagating along the $z$ axis towards $\pm z$, with $t$ the time and $k$ the wave vector.

[27] In practice, the helical mirror is placed on an $R^{\prime}=16 \mathrm{~mm}$ radius and $1 \mathrm{~mm}$ height axisymmetric seat made of the same material, which increases the pinning reproducibility of the triple contact line when performing experiments on distinct helical mirrors with various topological charges.

[28] Y. G. Ovseenko, Refined formulas for the resistance torque of an ellipsoid of revolution and a circular disk rotating in an infinite incompressible viscous fluid, Fluid Dyn. 4, 100 (1969).

[29] In the case of $\ell=1$ we use a two-ramp design with $\lambda / 4$ step height instead of a single-ramp design with $\lambda / 2$ step height in order to prevent drawbacks associated with the broken central symmetry of the mass distribution associated with the single-ramp design.

[30] M. Berry, Optical vortices evolving from helicoidal integer and fractional phase steps, J. Opt. 6, 259 (2004).

[31] K. Y. Bliokh and F. Nori, Transverse spin and surface waves in acoustic metamaterials, Phys. Rev. B 99, 020301 (2019).

[32] K. Y. Bliokh and F. Nori, Spin and orbital angular momenta of acoustic beams, Phys. Rev. B 99, 174310 (2019). 